Gut and Liver, Vol. 12, No. 6, November 2018, pp. 728-735

\title{
Efficacy of Nab-Paclitaxel Plus Gemcitabine and Prognostic Value of Peripheral Neuropathy in Patients with Metastatic Pancreatic Cancer
}

\author{
Min Su You, Ji Kon Ryu, Young Hoon Choi, Jin Ho Choi, Gunn Huh, Woo Hyun Paik, Sang Hyub Lee, and Yong-Tae Kim \\ Department of Internal Medicine and Liver Research Institute, Seoul National University College of Medicine, Seoul, Korea
}

Background/Aims: The combination of nab-paclitaxel and gemcitabine (nab-P/Gem) is widely used for treating metastatic pancreatic cancer (MPC). We aimed to evaluate the therapeutic outcomes and prognostic role of treatment-related peripheral neuropathy in patients with MPC treated with nab-P/Gem in clinical practice. Methods: MPC patients treated with nab-P/Gem as the first-line chemotherapy were included. All 88 Korean patients underwent at least two cycles of nab-P/Gem combination chemotherapy (125 and 1,000 $\mathrm{mg} / \mathrm{m}^{2}$, respectively). Treatment-related adverse events were monitored through periodic follow-ups. Overall survival and progression-free survival were estimated by the KaplanMeier method, and the Cox proportional hazards regression linear model was applied to assess prognostic factors. To evaluate the prognostic value of treatment-related peripheral neuropathy, the landmark point analysis was used. Results: Patients underwent a mean of $6.7 \pm 4.2$ cycles during $6.3 \pm 4.4$ months. The median overall survival and progression-free survival rates were 14.2 months (95\% confidence interval [Cl], 11.8 to 20.3 months) and 8.4 months $(95 \% \mathrm{Cl}, 7.1$ to 13.2 months), respectively. The disease control rate was 84.1\%; a partial response and stable disease were achieved in $30(34.1 \%)$ and $44(50.0 \%)$ patients, respectively. Treatment-related peripheral neuropathy developed in 52 patients (59.1\%), and $13(14.8 \%)$ and $16(18.2 \%)$ patients experienced grades 2 and 3 neuropathy, respectively. In the landmark model, at 6 months, treatment-related peripheral neuropathy did not have a significant correlation with survival $(p=0.089)$. Conclusions: $\mathrm{Nab}-\mathrm{P} / \mathrm{Gem}$ is a reasonable choice for treating MPC, as it shows a considerable disease control rate while the treatment-related peripheral neuropathy was tolerable. The prognostic role of treatment-related neuropathy was limited. (Gut Liver 2018;12:728-735)
Key Words: Pancreatic neoplasm; Metastasis; Chemotherapy; Gemcitabine; Nab-paclitaxel

\section{INTRODUCTION}

Pancreatic cancer, one of the most deadly malignancies, is now the fourth leading cause of cancer-related death and is expected to rank as the second leading cause by 2030., Most patients with pancreatic cancer are initially diagnosed at advanced stages since there are no adequate screening tests, and early stages lack specific symptoms. ${ }^{3}$ While curative surgery is attempted in some patients with locally advanced cancer invading adjacent vascular or lymphatic systems, ${ }^{4}$ only palliative or supportive care is possible in patients with metastatic pancreatic cancer (MPC). MPC has an extremely poor prognosis and a 5-year survival rate of $2.6 \% .^{5}$

The nab-paclitaxel plus gemcitabine (nab-P/Gem) regimen has been approved in many countries after the phase III MPACT trial, which reported that the addition of nab-paclitaxel to gemcitabine significantly increases overall survival (OS) with tolerable adverse events. ${ }^{6}$ Subsequent retrospective series have also stated that nab-P/Gem is an effective, well-tolerated, costeffective, and active regimen in pretreated patients. ${ }^{71}$ However, most retrospective studies about the efficacy and safety of nab$\mathrm{P} / \mathrm{Gem}$ are limited by a small number of patients. ${ }^{7,9,10}$

The major toxicity of nab-paclitaxel, a microtubule-stabilizing agent, is peripheral neuropathy. ${ }^{12,13}$ Recent retrospective studies have reported $30.4 \%$ to $56.8 \%$ incidence of peripheral neuropathy during the course of nab-P/Gem combination chemotherapy, raising questions regarding discrepancies between the safety profile of nab-paclitaxel in clinical trials and in realworld practice. ${ }^{6,711}$ Subset analysis ${ }^{14}$ of the MPACT trial and

Correspondence to: Ji Kon Ryu

Department of Internal Medicine and Liver Research Institute, Seoul National University Hospital, Seoul National University College of Medicine, 101 Daehak-ro, Jongno-gu, Seoul 03080, Korea

Tel: +82-2-2072-1962, Fax: +82-2-762-9662, E-mail: jkryu@snu.ac.kr

Received on May 16, 2018. Revised on June 23, 2018. Accepted on July 24, 2018. Published online October 29, 2018

pISSN 1976-2283 eISSN 2005-1212 https://doi.org/10.5009/gnl18220

@) This is an Open Access article distributed under the terms of the Creative Commons Attribution Non-Commercial License (http://creativecommons.org/licenses/by-nc/4.0) which permits unrestricted non-commercial use, distribution, and reproduction in any medium, provided the original work is properly cited. 
the report by Cho et al. ${ }^{11}$ demonstrated that the occurrence of treatment-related peripheral neuropathy independently predicts improved OS in patients with MPC treated with nab-P/Gem.

In post-hoc studies of the MPACT trial, several prognostic factors associated with OS of MPC were identified, including age, performance status, and liver metastasis. ${ }^{15,16}$ In addition, Luo et al. ${ }^{17}$ reported that baseline neutrophil-to-lymphocyte ratio (NLR) $\geq 3.1$ was a poor prognostic factor in patients with advanced pancreatic cancer. NLR is an indicator of systemic inflammatory response and is widely known as a prognostic factor in various carcinomas. However, there is a lack of studies investigating the prognostic role of NLR in patients with MPC who received nab-P/Gem combination chemotherapy.

Since the MPACT trial, nab-P/Gem combination therapy has been widely used in patients with MPC. However, clinical data regarding treatment outcomes and prognostic factors in realworld practice are still lacking. Subsequent studies show a high occurrence rate of adverse drug events, especially peripheral neuropathy, and an association with long-term prognosis. We aimed to evaluate the efficacy of nab-P/Gem combination therapy and investigate an independent prognostic role of treatment-related peripheral neuropathy.

\section{MATERIALS AND METHODS}

\section{Study subjects}

Medical records of 88 Korean patients treated with nab-P/ Gem as first-line chemotherapy for MPC at Seoul National University Hospital from June 2015 to October 2017 were reviewed. All patients were diagnosed with pancreatic adenocarcinoma by pathologic examination and were classified into metastatic disease. Metastasis to distant organs or lymph nodes was evaluated by pathologic findings and/or ${ }^{18} \mathrm{~F}$-fluoro-2-deoxy-D-glucose positron emission tomography with computed tomography. Patients who had previous cytotoxic chemotherapies were excluded. Informed consent was obtained from all patients undergoing chemotherapy. The Institutional Review Board of Seoul National University Hospital, Seoul, Korea, approved this study (1705132-856).

\section{Treatment and data collection}

All patients received at least two cycles of combination chemotherapy; each cycle comprised nab-paclitaxel at a dose of $125 \mathrm{mg} / \mathrm{m}^{2}$, followed by gemcitabine at a dose of $1,000 \mathrm{mg} /$ $\mathrm{m}^{2}$ on days 1,8 , and 15 every 4 weeks. The dose and treatment intervals were adjusted according to patient performance status and development of adverse events including peripheral neuropathy, neutropenia, and thrombocytopenia. If progression was confirmed, treatment with nab-P/Gem was discontinued. All patients were evaluated for initial tumor response after initial therapy except for two patents lost to follow-up.

Data regarding patient demographics, Eastern Cooperative
Oncology Group (ECOG) performance status, Charlson comorbidity index, primary tumor site, metastatic location, baseline and follow-up laboratory findings, further subsequent lines of therapy, reasons for treatment discontinuation, and history of previous treatment including curative surgery and biliary drainage procedures were collected. Baseline NLR was defined as absolute neutrophil count divided by absolute lymphocyte count in peripheral blood. To evaluate tumor response, contrast computed tomography, magnetic resonance imaging, or ${ }^{18} \mathrm{~F}-\mathrm{flu}$ orodeoxyglucose-positron emission tomography was performed every 8 to 12 weeks, at the discretion of the treating physician. The Response Evaluation Criteria in Solid Tumors guideline was used to assess tumor response, and the best overall response was evaluated. ${ }^{18}$ Mortality data were collected from the database established by the Korean Ministry of the Interior and Safety.

\section{Treatment outcome measurements}

The primary outcome measures were OS and progression-free survival (PFS). OS was determined from the date of first chemotherapy to death from any cause, whereas patients who were still alive were censored at the date of last known follow-up. PFS was calculated from the date of first chemotherapy to the earliest date of radiographic progression or death. Investigators monitored development of treatment-related adverse events, periodic laboratory tests, rates of dose reductions, and discontinuations of combination therapy. Treatment-related adverse events were graded according to the National Cancer Institute Common Terminology Criteria for Adverse Events, version 4.0 (CTCAE v4.0).

Treatment-related peripheral neuropathy during the course of combination chemotherapy was assessed for its association with prognosis. In addition, we evaluated factors associated with prognosis in patients with MPC. Baseline patient demographics and laboratory findings including blood cell count, transaminases, total bilirubin, estimated glomerular filtration rate, NLR, and carbohydrate antigen 19-9 (CA 19-9) were reviewed.

\section{Statistical analysis}

For baseline characteristics, numerical data were expressed as means and standard deviation or medians and range depending on distributions of the data. Survival analysis was based on the Kaplan-Meier method with median and 95\% confidence interval (CI). Univariable and multivariable analyses were performed by Cox proportional-hazard model to reveal the prognostic factors related to OS. Hazard ratio (HR) for increased risk of mortality and 95\% CI were calculated. Multivariable analysis was performed using factors with $\mathrm{p}$-values $<0.1$ in the univariable analysis. p-values $\leq 0.05$ were considered statistically significant in the final multivariable analysis model. The landmark point method was used to analyze the association between peripheral neuropathy and prognosis to minimize lead-time bias. ${ }^{19}$ All statistical computations were performed using $\mathrm{R}$ version 3.3.3 
for Windows (Institute for Statistics and Mathematics, Vienna, Austria; http://www.R-project.org).

\section{RESULTS}

\section{Clinical characteristics of patients}

Baseline patient characteristics are shown in Table 1. The

Table 1. Baseline Characteristics

\begin{tabular}{|c|c|}
\hline Characteristic & Patients with MPC $(\mathrm{n}=88)$ \\
\hline Age, yr & $61.5(32.0-83.0)$ \\
\hline \multicolumn{2}{|l|}{ Sex } \\
\hline Female & $33(37.5)$ \\
\hline Male & $55(62.5)$ \\
\hline \multicolumn{2}{|l|}{ ECOG } \\
\hline $0-1$ & 85 (96.6) \\
\hline 2 & $3(3.0)$ \\
\hline Charlson comorbidity index & $8.5 \pm 1.3$ \\
\hline \multicolumn{2}{|l|}{ Primary tumor location } \\
\hline Head & $39(44.3)$ \\
\hline Body and tail & $49(55.7)$ \\
\hline \multicolumn{2}{|l|}{ Metastasis } \\
\hline Liver & $55(62.5)$ \\
\hline Lung & $15(17.0)$ \\
\hline Peritoneum & $27(30.7)$ \\
\hline Distant LNs & $12(13.6)$ \\
\hline \multicolumn{2}{|l|}{ No. of metastasis } \\
\hline 1 & $57(64.8)$ \\
\hline 2 & $21(23.9)$ \\
\hline 3 or more & $10(11.4)$ \\
\hline \multicolumn{2}{|l|}{ Previous therapy } \\
\hline Bile duct stent & $15(18.1)^{*}$ \\
\hline Curative surgery & $17(19.3)$ \\
\hline \multicolumn{2}{|l|}{ Baseline laboratory findings } \\
\hline Hemoglobin, g/dL & $12.5 \pm 1.5$ \\
\hline Platelet, $10^{3} / \mu \mathrm{L}$ & $228.5 \pm 73.6$ \\
\hline ANC, cells $/ \mu \mathrm{L}$ & $4,531.9 \pm 2,584.0$ \\
\hline AST, IU/L & $31.6 \pm 25.9$ \\
\hline ALT, IU/L & $37.4 \pm 50.4$ \\
\hline Total bilirubin, mg/dL & $0.8 \pm 0.8$ \\
\hline $\mathrm{eGFR}, \mathrm{mL} / \mathrm{min} / 1.73 \mathrm{~m}^{2}$ & $95.8 \pm 19.9$ \\
\hline NLR & $3.1 \pm 2.3$ \\
\hline CA 19-9, U/mL & $473.0(1.7-5,322.5)^{\dagger}$ \\
\hline
\end{tabular}

Data are presented as median (range), number (\%), or mean \pm SD. MPC, metastatic pancreatic cancer; ECOG, Eastern Cooperative Oncology Group; LN, lymph node; ANC, absolute neutrophil count; AST, aspartate aminotransferase; ALT, alanine aminotransferase; eGFR, estimated glomerular filtration rate; NLR, neutrophil-to-lymphocyte ratio; CA 19-9, carbohydrate antigen 19-9.

*Includes two patients treated by percutaneous approach; ${ }^{\dagger}$ Median (interquartile range). median age was 61.5 years, and 23 patients (26.1\%) were older than 70 years. Most patients ( $\mathrm{n}=85,96.6 \%)$ had ECOG performance status scores of 0 or 1 . Mean Charlson score was $8.5 \pm 1.3$, and it was 9 or more in 14 patients (15.9\%). Metastasis was most commonly identified in the liver in 55 patients (62.5\%), followed by the peritoneum in 27 patients (30.7\%) and lung in 15 patients (17.0\%). There were 31 patients (35.2\%) with metastasis at two or more sites, while 57 patients (64.8\%) had one metastatic site. Seventeen patients (19.3\%) had a history of curative surgery before diagnosis of MPC.

\section{Treatment course and adverse events}

Table 2 displays the clinical course and treatment outcomes during the course of combination chemotherapy. The median follow-up duration was 10.4 months (range, 1.5 to 21.5 months). Patients received $6.7 \pm 4.2$ total cycles of treatment. Median OS and PFS were 14.2 months (95\% CI, 11.8 to 20.3 months) and 8.4 months (95\% CI, 7.1 to 13.2 months), respectively (Fig. 1). Tumor control was achieved in 74 patients (84.1\%). Overall, partial response was achieved in 30 patients (34.1\%), and stable disease was achieved in 44 patients (50.0\%). No patient achieved complete response. Of the patients with progressive disease, further regimens were employed in 43 (48.9\%). Among these, combination of folinic acid, fluorouracil, irinotecan, and oxaliplatin was the most common regimen, used in 16 patients (37.2\%).

Adverse drug events over grade 2 are summarized in Table 3. Several adverse events over grade 3 were observed, including neutropenia in 34 patients (38.6\%), anemia in 19 patients

Table 2. Treatment Data during the Course of Nab-Paclitaxel/Gem Combination Chemotherapy

\begin{tabular}{lc}
\hline \multicolumn{1}{c}{ Variable } & Value \\
\hline Total cycle & $6.7 \pm 4.2$ \\
Treatment duration, mo & $6.3 \pm 4.4$ \\
Cumulative dose & \\
Nab-P, $\mathrm{mg} / \mathrm{m}^{2}$ & $1,812.8 \pm 1,040.3$ \\
Gem, $\mathrm{mg} / \mathrm{m}^{2}$ & $14,679.5 \pm 8,442.5$ \\
Relative dose per cycle & \\
Nab-P, $\%$ & $94.9 \pm 24.2$ \\
Gem, \% & $95.8 \pm 24.2$ \\
Overall best response & \\
Partial response & $30(34.1)$ \\
Stable disease & $44(50.0)$ \\
Progression & $12(13.6)$ \\
Not evaluable & $2(2.3)$ \\
OS, mo & $14.2(11.8-20.3)$ \\
PFS, mo & $8.4(7.1-13.2)$
\end{tabular}

Data are presented as mean $\pm \mathrm{SD}$, number $(\%)$, or median $(95 \%$ confidence interval).

Nab-P, nab-paclitaxel; Gem, gemcitabine; OS, overall survival; PFS, progression-free survival. 
(21.6\%), and peripheral neuropathy in 16 patients (18.2\%). Febrile neutropenia occurred in 5 patients (5.7\%). The most common adverse events that caused dose reduction and discontinuation of therapy were peripheral neuropathy of grade 1 or $2(19.3 \%)$ and grade $3(18.2 \%)$. Overall, dose reduction was needed in 39 patients (44.3\%) for nab-paclitaxel and 28 (31.8\%) patients for gemcitabine. After three cycles of therapy, 44 patients (50.0\%) underwent dose reduction $(n=21,23.9 \%)$ or discontinued treatment $(\mathrm{n}=23,26.1 \%)$.

\section{Peripheral neuropathy during chemotherapy}

Treatment-related peripheral neuropathy occurred in 52 patients (59.1\%) during the total cycle. The mean number of cycles until developing grade 1,2 , and 3 peripheral neuropathy was $2.8 \pm 1.7,4.7 \pm 2.6$, and $6.2 \pm 3.2$, respectively. Most peripheral neuropathy began as grade 1 , and 46 patients (52.3\%) experienced

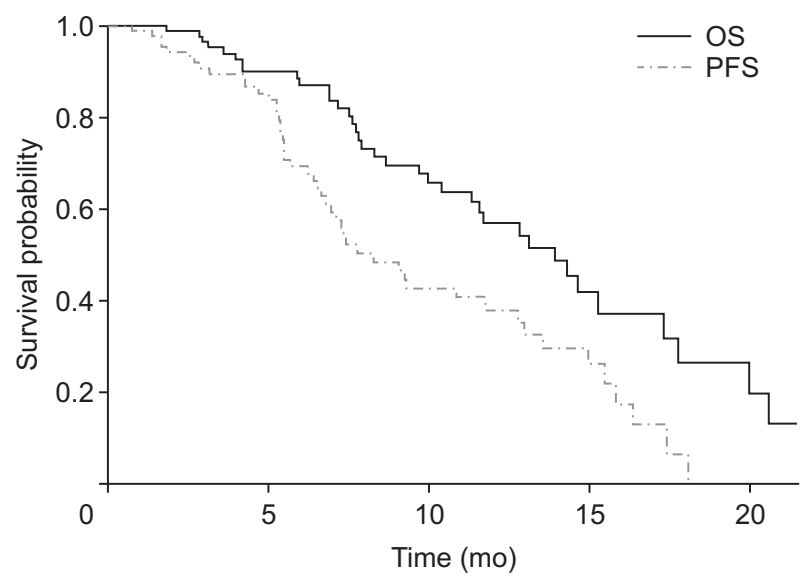

Fig. 1. OS and PFS graphed by the Kaplan-Meier survival method. OS, overall survival; PFS, progression-free survival. grade 1 peripheral neuropathy. Worsening of grade 1 peripheral neuropathy to grade 2 or 3 was observed in 25 patients (54.3\%), and worsening of grade 2 to grade 3 peripheral neuropathy was observed in 13 patients (54.2\%). In 24 patients who experienced grade 2 peripheral neuropathy, dose reduction or delay was required in 16 (66.7\%). All patients with grade 3 peripheral neuropathy had to discontinue combination chemotherapy, and the time to recovery from neuropathy after discontinuation was 66.0 (interquartile range [IQR], 35.0 to 102.0) days.

The prognostic value of peripheral neuropathy was assessed by the landmark point method, which was determined based on survival status at 6 months after initiation of therapy (Table 4). In the naïve model, patients with peripheral neuropathy had a significantly lower hazard ratio and longer survival. In the landmark model at 6 months, treatment-related peripheral neuropathy did not have a significant correlation with survival. There were 11 deaths (57.9\%) in 19 patients with peripheral neuropathy and 17 deaths (43.6\%) in 39 patients without peripheral

Table 3. Treatment-Related Adverse Events

\begin{tabular}{lccc}
\hline \multicolumn{1}{c}{ Complications } & Grade 2 & Grade 3 & Grade 4 \\
\hline Febrile neutropenia & - & $5(5.7)$ & - \\
Anemia & $38(43.2)$ & $19(21.6)$ & - \\
Thrombocytopenia & $7(8.0)$ & $3(3.4)$ & $2(2.3)$ \\
Neutropenia & $22(25.0)$ & $20(22.7)$ & $14(15.9)$ \\
Peripheral neuropathy* & $13(14.8)$ & $16(18.2)$ & - \\
Peripheral edema & $17(19.3)$ & $4(4.5)$ & - \\
Diarrhea & $3(3.4)$ & $1(1.1)$ & - \\
Nausea, vomiting & $15(17.0)$ & $3(3.4)$ & - \\
\hline
\end{tabular}

Data are presented as number (\%).

${ }^{*}$ Grade 1 peripheral neuropathy developed in 23 patents (26.1\%).

Table 4. Results of Landmark Analysis for Overall Survival by Survival Status at 6 Months after Initiation of Therapy

\begin{tabular}{|c|c|c|c|c|c|c|}
\hline \multirow[b]{2}{*}{ Variables } & \multicolumn{3}{|c|}{ Naïve model } & \multicolumn{3}{|c|}{ Landmark set at 6 mo } \\
\hline & $\begin{array}{c}\text { Patients without } \\
\text { neuropathy }(n=36)\end{array}$ & $\begin{array}{c}\text { Patients with } \\
\text { neuropathy }(n=52)\end{array}$ & $\mathrm{p}$-value & $\begin{array}{c}\text { Patients without } \\
\text { neuropathy }(n=19)\end{array}$ & $\begin{array}{c}\text { Patients with } \\
\text { neuropathy }(n=39)\end{array}$ & p-value \\
\hline OS (95\% CI), mo & 10.13 (7.87-NE) & $15.53(13.3-\mathrm{NE})$ & 0.007 & $11.4(9.7-\mathrm{NE})$ & $15.3(13.2-\mathrm{NE})$ & 0.089 \\
\hline Death & $17(47.2)$ & $19(36.5)$ & & 11 (57.9) & $17(43.6)$ & \\
\hline Adjusted HR (95\% CI)* & 1.00 & $0.34(0.17-0.67)$ & 0.002 & 1.00 & $0.43(0.20-0.94)$ & 0.034 \\
\hline Clinical benefit $^{\dagger}$ & & & $<0.001$ & & & 0.006 \\
\hline No & $13(36.1)$ & $1(1.9)$ & & $6(31.6)$ & $1(2.6)$ & \\
\hline Yes & $23(63.9)$ & $51(98.1)$ & & $13(68.4)$ & $38(97.4)$ & \\
\hline \multicolumn{7}{|l|}{ Total accumulate dose } \\
\hline nab-P, $10^{3} \mathrm{mg} / \mathrm{m}^{2}$ & $1.3 \pm 0.9$ & $2.2 \pm 1.0$ & $<0.001$ & $1.8 \pm 1.0$ & $2.4 \pm 1.0$ & 0.029 \\
\hline Gem, $10^{3} \mathrm{mg} / \mathrm{m}^{2}$ & $10.7 \pm 7.3$ & $17.5 \pm 8.0$ & $<0.001$ & $14.2 \pm 8.4$ & $19.7 \pm 8.0$ & 0.020 \\
\hline Total cycle & $5.1 \pm 3.9$ & $7.9 \pm 4.1$ & 0.002 & $6.9 \pm 4.4$ & $8.6 \pm 4.3$ & 0.174 \\
\hline Duration of treatment, mo & $4.8 \pm 4.3$ & $7.4 \pm 4.7$ & 0.008 & $6.8 \pm 4.8$ & $8.0 \pm 4.3$ & 0.373 \\
\hline
\end{tabular}

Data are presented as number (\%) or mean \pm SD.

OS, overall survival; CI, confidence interval; NE, not evaluable; HR, hazard ratio; nab-P, nab-paclitaxel; Gem, gemcitabine.

${ }^{*}$ Adjusted for neutrophil-to-lymphocyte (NLR) ratio $>3.1$ and the presence of liver metastasis; ${ }^{\dagger}$ Includes partial response and stable disease. 
neuropathy. Median OS in patient without and with peripheral neuropathy was 11.4 (95\% CI, 9.7 to not estimable [NE]) and 15.3 (95\% CI, 13.2 to NE) months, respectively ( $p=0.089)$. While adjusted HR was significant in the landmark model at 6 months, it was $0.50(95 \%$ CI, 0.17 to $1.49 ; \mathrm{p}=0.212)$ in the landmark model at 9 months and was not statistically significant. Like-

Table 5. Univariate and Multivariate Analyses by Cox Regression

\begin{tabular}{|c|c|c|c|c|}
\hline \multirow{2}{*}{ Variables } & \multicolumn{2}{|c|}{ Univariable analysis } & \multicolumn{2}{|c|}{ Multivariable analysis } \\
\hline & HR $(95 \%$ CI) & p-value & HR (95\% CI) & p-value \\
\hline \multicolumn{5}{|l|}{ Age $\geq 70$ yr } \\
\hline No & 1.00 & & & \\
\hline Yes & $0.87(0.39-1.94)$ & 0.729 & & \\
\hline \multicolumn{5}{|l|}{ Male } \\
\hline No & 1.00 & & & \\
\hline Yes & $0.86(0.44-1.72)$ & 0.678 & & \\
\hline \multicolumn{5}{|l|}{ ECOG } \\
\hline 0 & 1.00 & & & \\
\hline $1-2$ & $1.74(0.86-3.51)$ & 0.122 & & \\
\hline \multicolumn{5}{|l|}{ Charlson score $\geq 9$} \\
\hline No & 1.00 & & & \\
\hline Yes & $1.21(0.60-2.42)$ & 0.597 & & \\
\hline \multicolumn{5}{|c|}{ Primary tumor location } \\
\hline Body and tail & 1.00 & & & \\
\hline Head & $1.34(0.69-2.62)$ & 0.389 & & \\
\hline \multicolumn{5}{|l|}{ Metastasis } \\
\hline Liver & $2.49(1.13-5.50)$ & 0.023 & $2.03(0.91-4.53)$ & 0.084 \\
\hline Lung & $0.75(0.30-1.85)$ & 0.533 & & \\
\hline Peritoneum & $1.11(0.56-2.20)$ & 0.767 & & \\
\hline Distant LNs & $0.82(0.30-2.22)$ & 0.700 & & \\
\hline \multicolumn{5}{|l|}{ No. of metastasis } \\
\hline 1 & 1.00 & & & \\
\hline 2 & $1.41(0.63-3.13)$ & 0.401 & & \\
\hline 3 or more & $1.55(0.64-3.77)$ & 0.329 & & \\
\hline \multicolumn{5}{|l|}{ Previous therapy } \\
\hline Bile duct stent & $0.55(0.19-1.55)$ & 0.257 & & \\
\hline Curative surgery & $1.13(0.53-2.42)$ & 0.745 & & \\
\hline \multicolumn{5}{|c|}{ Total bilirubin $\geq 1.5$ ULN } \\
\hline No & 1.00 & & & \\
\hline Yes & $0.96(0.23-4.04)$ & 0.955 & & \\
\hline \multicolumn{5}{|c|}{ Transaminase $\geq 1.5$ ULN } \\
\hline No & 1.00 & & & \\
\hline Yes & $1.21(0.49-2.97)$ & 0.673 & & \\
\hline \multicolumn{5}{|l|}{$N L R \geq 3.1$} \\
\hline No & 1.00 & & 1.00 & \\
\hline Yes & $2.54(1.32-4.91)$ & 0.005 & $2.00(1.17-4.22)$ & 0.045 \\
\hline \multicolumn{5}{|l|}{ CA19-9 $\geq 1.5$ ULN } \\
\hline No & 1.00 & & 1.00 & \\
\hline Yes & $2.33(1.02-5.33)$ & 0.045 & $1.80(0.77-4.22)$ & 0.178 \\
\hline
\end{tabular}

HR, hazard ratio; CI, confidence interval; ECOG, Eastern Cooperative Oncology Group; LN, lymph node; ULN, upper limit of normal; NLR, neutrophil-to-lymphocyte ratio; CA 19-9, carbohydrate antigen 19-9. 
wise, clinical benefit rate was not significant in the landmark model at 9 months $(\mathrm{p}=0.062)$.

\section{Prognostic factors}

There were six patients (6.8\%) with baseline hyperbilirubinemia ( $\geq 1.5 \times \mathrm{ULN})$ due to malignant biliary obstruction; median bilirubin level was $2.9 \mathrm{mg} / \mathrm{dL}$ (IQR, 2.3 to $3.4 \mathrm{mg} / \mathrm{dL}$ ). Baseline abnormal level of bilirubin in all six patients with hyperbilirubinemia recovered to normal level during the course of combination chemotherapy, and the median duration to recovery was 25.0 days (IQR, 13.0 to 51.3 days). Baseline hyperbilirubinemia was not related to OS in univariable or multivariable analysis.

Possible prognostic factors related to OS are shown in Table 5. Univariable analysis demonstrated several meaningful factors associated with poor OS: metastasis to liver (HR, 2.49; 95\% CI, 1.13 to 5.50; $\mathrm{p}=0.023)$, CA19-9 $\geq 1.5 \times$ ULN (HR, 2.33; 95\% CI, 1.02 to $5.33 ; \mathrm{p}=0.045)$, and $\mathrm{NLR} \geq 3.1$ (HR, 2.54; 95\% CI, 1.32 to $4.91 ; \mathrm{p}=0.005$ ). In multivariable analysis, $\mathrm{NLR} \geq 3.1$ was an independent prognostic factor for poor OS (HR, 2.00; 95\% CI, 1.17 to $4.22 ; \mathrm{p}=0.045$ ). Median OS in patients with $\mathrm{NLR} \geq 3.1$ and patients with NLR<3.1 were 10.6 months (95\% CI, 7.3 to NE months) and 15.5 months (95\% CI, 14.2 to NE months), respectively (Fig. 2).

\section{DISCUSSION}

The objective of this study was to investigate the therapeutic outcomes, prognostic factors, and treatment-related adverse events in patients with MPC treated with nab-P/Gem as firstline chemotherapy. The results of this single-center retrospective study showed longer OS and PFS than the previous randomized controlled trial and the extension study of the MPACT trial based on mortality data from 90\% of enrolled patients. ${ }^{6,20}$ The reason for longer OS and PFS than other studies may stem from the restricted patient selection. In this study, total cycles

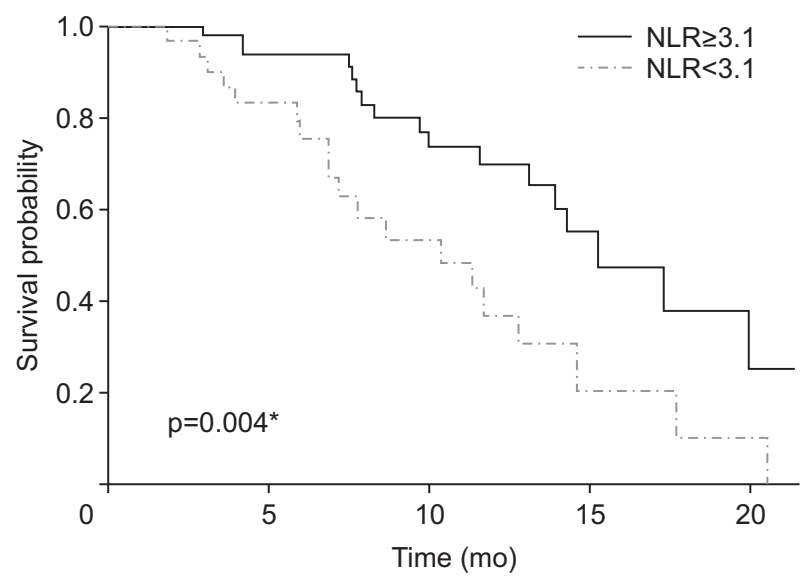

Fig. 2. Kaplan-Meier survival curves classified by baseline NLR $\geq 3$.1 or NLR <3.1.

NLR, neutrophil-to-lymphocyte ratio. ${ }^{*} \mathrm{p}<0.05$. and treatment duration were longer than in the MPACT trial, which had treatment duration of 3.9 months (range, 1.0 to 21.9 months), and another retrospective study with four median cycles. ${ }^{6,21}$ Meanwhile, a recent clinical trial in Japan reported an OS of 13.5 months (95\% CI, 10.6 to NE months) in patients with MPC. Another study based on survival data of the Korean population showed OS of 12.1 months (95\% CI, 10.7 to NE months), ${ }^{11,22}$ which is different from the previous result of the MPACT trial. The OS of our study was consistent with the two previous studies in Asia. Geographic or genetic factors may contribute to the therapeutic effect of nab-paclitaxel and gemcitabine combination therapy.

Tumor control rate in this study is in general agreement with other retrospective studies ranging from $43.5 \%$ to $81.0 \%$, ${ }^{9,10,21}$ which vary depending on whether they include pretreated patients, patients with liver function test abnormalities, and patients with advanced pancreatic cancer or only patients with metastatic pancreatic cancer. Since nab-P/Gem can pharmacokinetically cause hepatic impairment, care must be taken in patients with hepatic impairment and hyperbilirubinemia, and those patients should be monitored frequently. ${ }^{23}$ Results of this study demonstrated that elevated bilirubin and transaminases were not significantly related to OS, suggesting that abnormal laboratory findings caused by malignant biliary obstruction and liver metastasis in patients with MPC are not an absolute contraindication for chemotherapy, but rather an integral part of the inclusion criteria.

There was a difference in the frequency of peripheral neuropathy compared with other studies. In the MPACT trial, grade 3 peripheral neuropathy was reported in 17\% of patients; $10 \%$ of these patients required dose reduction, and 8\% required discontinuation of chemotherapy. ${ }^{6}$ In several retrospective studies, the incidence of grade 3 peripheral neuropathy ranged from $10.3 \%$ to $30.4 \% .^{7,9,21}$ In our study, $19.3 \%$ and $18.2 \%$ of all patients needed dose reduction and discontinuation of treatment due to peripheral neuropathy, respectively. Severe neuropathy was the most common drug-related adverse event that resulted in discontinuation of therapy, and the percentage of patients who had to stop treatment was almost twice as high as in the previous clinical trial. Nonetheless, the proportion of patients with grade 3 peripheral neuropathy was lower in this study than in the MPACT trial. All patients with grade 3 neuropathy in our study had to discontinue the combination drug. Although most studies have assessed peripheral neuropathy based on CTCAE, there are some difficulties in objectively grading peripheral neuropathy in practice, and differences exist between studies. Studies regarding an objective grading system for treatment-related peripheral neuropathy are required.

Peripheral neuropathy is the main adverse effect of nab-paclitaxel and is reported in 51.0\% of cancer patients who receive nab-paclitaxel. ${ }^{24}$ In breast cancer, studies to identify factors related to treatment-related peripheral neuropathy have been 
actively performed $;^{25}$ however, studies on pancreatic cancer are still lacking. In the subset analysis of MPACT trial, long-term administration of nab-paclitaxel was associated with treatmentrelated peripheral neuropathy, and other retrospective study revealed no risk factors. ${ }^{11,14}$ We also analyzed the association between treatment-related peripheral neuropathy and baseline laboratory findings, tumor characteristics, and patient demographics including ages, sex, and comorbidity index, and no risk factors were found. Further investigations to identify factors associated with peripheral neuropathy in patients with MPC treated with nab-P/Gem are warranted.

In general, the symptoms of neuropathy can be controlled by reducing the dose of nab-paclitaxel or increasing the interval between doses. ${ }^{6}$ In this study, peripheral neuropathy was manageable for most patients with dose reduction or discontinuation, coinciding well with previous studies. However, because more than half of patients with grade 1 peripheral neuropathy ultimately progress to a worse grade, dose reduction should be considered in the early state of neuropathy. Although about half of patients had to reduce their dose during the course of treatment in this study, the clinical benefit rate was significantly high. These findings coincide well with other studies that indicate the effectiveness of a biweekly regimen and reduced agent doses. These results suggest that the current standard therapeutic dose and schedule may need adjustment. ${ }^{26,27}$ Since previous studies did not have a comparative design, further studies are warranted regarding the appropriate dose and interval.

NLR is a prognostic factor for various tumors, including advanced pancreatic cancer in patients who undergo chemotherapy. Neutrophils secrete vascular endothelial growth factor to promote and mediate the development and proliferation of cancer. ${ }^{28}$ In this study, the results of multivariable analysis demonstrated that baseline NLR is an independent prognostic predictor for patients with MPC undergoing nab-P/Gem combination chemotherapy. Because cancer-associated inflammation is associated with cancer prognosis, many studies have attempted to target inflammation for treatment. Recently, drugs targeting specific cytokines and chemokines have been studied. ${ }^{29,30}$ As a measure of cancer-associated inflammation, the role of NLR as an important predictor of prognosis should be emphasized in future studies.

In this study, OS and PFS were based on relatively few deaths (36/88, 40.9\%), compared to the MPACT trial (692/861, 80.4\%). However, the aim of this study to evaluate the effectiveness of nab-P/Gem was not constrained because OS and PFS were in close agreement with the results of other studies. Second, this study has limitations due to its small sample size and statistical power. This study set the landmark point at 6 months, and it confirmed that the OS and peripheral neuropathy were not significantly correlated, whereas adjusted HR and clinical benefit rate still showed significant difference between two groups. In further analysis with landmark point at 9 months, the statistical differences clearly diminished, indicating statistical bias may stem from the small sample size. Unlike previous studies that evaluated prognostic role of treatment-related peripheral neuropathy in cross-sectional analysis, this study has advantages in that is used statistical model to reduce lead-time bias. ${ }^{11,14}$

Combination chemotherapy of nab-P/Gem in patients with MPC showed similar disease control rate and comparable rate of treatment-related peripheral neuropathy as compared to previous studies, and the neuropathy was not a predictor of prognosis. This regimen is a reasonable choice for palliative treatment. Careful attention and early interventions, such as dose reduction or delay, are required in patients with treatment-related peripheral neuropathy.

\section{CONFLICTS OF INTEREST}

No potential conflict of interest relevant to this article was reported.

\section{ACKNOWLEDGEMENTS}

This study was supported by Seoul National University Hospital Research fund (grant no. 04-2015-3020).

\section{REFERENCES}

1. Siegel RL, Miller KD, Jemal A. Cancer statistics, 2016. CA Cancer J Clin 2016;66:7-30

2. Rahib L, Smith BD, Aizenberg R, Rosenzweig AB, Fleshman JM, Matrisian LM. Projecting cancer incidence and deaths to 2030: the unexpected burden of thyroid, liver, and pancreas cancers in the United States. Cancer Res 2014;74:2913-2921.

3. Manji GA, Olive KP, Saenger YM, Oberstein P. Current and emerging therapies in metastatic pancreatic cancer. Clin Cancer Res 2017;23:1670-1678.

4. Abou-Khalil J, Rocha FG. Surgical strategies and novel therapies for locally advanced pancreatic cancer. J Surg Oncol 2017;116:1624.

5. Vogel A, Römmler-Zehrer J, Li JS, McGovern D, Romano A, Stahl M. Efficacy and safety profile of nab-paclitaxel plus gemcitabine in patients with metastatic pancreatic cancer treated to disease progression: a subanalysis from a phase 3 trial (MPACT). BMC Cancer 2016;16:817.

6. Von Hoff DD, Ervin T, Arena FP, et al. Increased survival in pancreatic cancer with nab-paclitaxel plus gemcitabine. N Engl J Med 2013;369:1691-1703.

7. Ottaiano A, Capozzi M, De Divitiis C, et al. Nab-paclitaxel and gemcitabine in advanced pancreatic cancer: the one-year experience of the National Cancer Institute of Naples. Anticancer Res 2017;37:1975-1978.

8. Carrato A, García P, López R, et al. Cost-utility analysis of nanoparticle albumin-bound paclitaxel (nab-paclitaxel) in combi- 
nation with gemcitabine in metastatic pancreatic cancer in Spain: results of the PANCOSTABRAX study. Expert Rev Pharmacoecon Outcomes Res 2015;15:579-589.

9. Bertocchi P, Abeni C, Meriggi F, et al. Gemcitabine plus nabpaclitaxel as second-line and beyond treatment for metastatic pancreatic cancer: a single institution retrospective analysis. Rev Recent Clin Trials 2015;10:142-145.

10. Zhang Y, Hochster H, Stein S, Lacy J. Gemcitabine plus nab-paclitaxel for advanced pancreatic cancer after first-line FOLFIRINOX: single institution retrospective review of efficacy and toxicity. Exp Hematol Oncol 2015;4:29.

11. Cho IR, Kang H, Jo JH, et al. Efficacy and treatment-related adverse events of gemcitabine plus nab-paclitaxel for treatment of metastatic pancreatic cancer "in a Korean" population: a singlecenter cohort study. Semin Oncol 2017;44:420-427.

12. Lee JJ, Swain SM. Peripheral neuropathy induced by microtubulestabilizing agents. J Clin Oncol 2006;24:1633-1642.

13. Dumontet C, Jordan MA. Microtubule-binding agents: a dynamic field of cancer therapeutics. Nat Rev Drug Discov 2010;9:790-803.

14. Goldstein D, Von Hoff DD, Moore M, et al. Development of peripheral neuropathy and its association with survival during treatment with nab-paclitaxel plus gemcitabine for patients with metastatic adenocarcinoma of the pancreas: a subset analysis from a randomised phase III trial (MPACT). Eur J Cancer 2016;52:85-91.

15. Tabernero J, Chiorean EG, Infante JR, et al. Prognostic factors of survival in a randomized phase III trial (MPACT) of weekly nabpaclitaxel plus gemcitabine versus gemcitabine alone in patients with metastatic pancreatic cancer. Oncologist 2015;20:143-150.

16. Kunzmann V, Ramanathan RK, Goldstein D, et al. Tumor reduction in primary and metastatic pancreatic cancer lesions with nabpaclitaxel and gemcitabine: an exploratory analysis from a phase 3 study. Pancreas 2017;46:203-208.

17. Luo G, Guo M, Liu Z, et al. Blood neutrophil-lymphocyte ratio predicts survival in patients with advanced pancreatic cancer treated with chemotherapy. Ann Surg Oncol 2015;22:670-676.

18. Eisenhauer EA, Therasse P, Bogaerts J, et al. New response evaluation criteria in solid tumours: revised RECIST guideline (version 1.1). Eur J Cancer 2009;45:228-247.

19. Jones M, Fowler R. Immortal time bias in observational studies of time-to-event outcomes. J Crit Care 2016;36:195-199.
20. Goldstein D, El-Maraghi RH, Hammel P, et al. nab-paclitaxel plus gemcitabine for metastatic pancreatic cancer: long-term survival from a phase III trial. J Natl Cancer Inst 2015;107:dju413.

21. Lo Re G, Santeufemia DA, Foltran L, Bidoli E, Basso SM, Lumachi F. Prognostic factors of survival in patients treated with nabpaclitaxel plus gemcitabine regimen for advanced or metastatic pancreatic cancer: a single institutional experience. Oncotarget 2015;6:8255-8260.

22. Ueno H, Ikeda M, Ueno M, et al. Phase I/II study of nab-paclitaxel plus gemcitabine for chemotherapy-naïve Japanese patients with metastatic pancreatic cancer. Cancer Chemother Pharmacol 2016; 77:595-603.

23. Vogel A, Kullmann F, Kunzmann V, et al. Patients with advanced pancreatic cancer and hyperbilirubinaemia: review and German expert opinion on treatment with nab-paclitaxel plus gemcitabine. Oncol Res Treat 2015;38:596-603.

24. Peng L, Bu Z, Ye X, Zhou Y, Zhao Q. Incidence and risk of peripheral neuropathy with nab-paclitaxel in patients with cancer: a meta-analysis. Eur J Cancer Care (Engl) 2017;26:e12407.

25. Rivera E, Cianfrocca M. Overview of neuropathy associated with taxanes for the treatment of metastatic breast cancer. Cancer Chemother Pharmacol 2015;75:659-670.

26. Ahn DH, Krishna K, Blazer M, et al. A modified regimen of biweekly gemcitabine and nab-paclitaxel in patients with metastatic pancreatic cancer is both tolerable and effective: a retrospective analysis. Ther Adv Med Oncol 2017;9:75-82.

27. Scheithauer W, Ramanathan RK, Moore M, et al. Dose modification and efficacy of nab-paclitaxel plus gemcitabine vs. gemcitabine for patients with metastatic pancreatic cancer: phase III MPACT trial. J Gastrointest Oncol 2016;7:469-478.

28. Kusumanto YH, Dam WA, Hospers GA, Meijer C, Mulder NH. Platelets and granulocytes, in particular the neutrophils, form important compartments for circulating vascular endothelial growth factor. Angiogenesis 2003;6:283-287.

29. Matsuo Y, Takeyama H, Guha S. Cytokine network: new targeted therapy for pancreatic cancer. Curr Pharm Des 2012;18:24162419.

30. Yoshie 0. Chemokine receptors as therapeutic targets. Nihon Rinsho Meneki Gakkai Kaishi 2013;36:189-196. 